\title{
Differential Organ-Specific Tumor Response to Immune Checkpoint Inhibitors in Hepatocellular Carcinoma
}

\author{
Li-Chun Lü, c, h Chiun Hsu ${ }^{a, c}$ Yu-Yun Shao a, c, h Yee Chao ${ }^{f}$ \\ Chia-Jui Yeng I-Lun Shih ${ }^{d}$ Yi-Ping Hung ${ }^{f}$ Chun-Jung Chang ${ }^{a}{ }^{c}$ \\ Ying-Chun Shen ${ }^{a, c}, h$ Jhe-Cyuan Guo ${ }^{b, c, h}$ Tsung-Hao Liu ${ }^{a, c, i}$ \\ Chih-Hung Hsu ${ }^{a, c, h} \quad$ Ann-Lii Chenga, c,e, h \\ ${ }^{a}$ Graduate Institute of Oncology, National Taiwan University College of Medicine, \\ Taipei, Taiwan; ${ }^{b}$ Graduate Institute of Clinical Medicine, National Taiwan University College of \\ Medicine, Taipei, Taiwan; ' Departments of Oncology, National Taiwan University Hospital, \\ Taipei, Taiwan; ${ }^{d}$ Department of Medical Imaging, National Taiwan University Hospital,

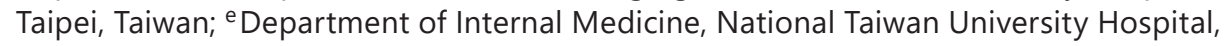

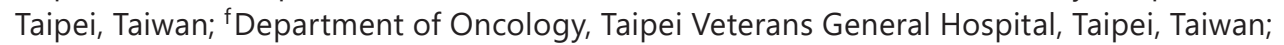 \\ 9Department of Internal Medicine, National Cheng Kung University Hospital, \\ Tainan, Taiwan; ${ }^{\text {h}}$ National Taiwan University Cancer Center, Taipei, Taiwan; ' Department of \\ Internal Medicine, National Taiwan University Hospital Hsinchu Branch, Hsinchu, Taiwan
}

\section{Keywords}

Hepatocellular carcinoma - Immune checkpoint inhibitor - Tumor microenvironment - Tumor response $\cdot$ Immune contexture

\begin{abstract}
Background and Aims: Immune checkpoint inhibitors (ICls) exhibit significant clinical activity in patients with advanced hepatocellular carcinoma (HCC). This study explored whether tumor response to ICls in HCC varies among different organs. Methods: We reviewed the data of patients with advanced $\mathrm{HCC}$ who had received ICls. Patients with measurable diseases were enrolled. Organ-specific response criteria, adapted from RECIST 1.1 and immune-related RECIST, were used to evaluate the objective response to ICls in tumors located in the liver, lung, lymph node, and other intra-abdominal sites. Results: Of the 75 enrolled patients with advanced HCC, 51 and 11 patients had chronic hepatitis B virus and chronic hepatitis $C$ virus infection, respectively. Regarding $\mathrm{ICI}$ treatment, 58, 1, and 16 patients had undergone anti-PD-1/ anti-PD-L1 monoclonal antibody (mAb) alone, anti-CTLA4 mAb alone, and anti-PD-1 mAb plus anti-CTLA4 mAb, respectively; 20 and 55 patients had received ICls as first-line or $\geq$ second-line therapy. The overall objective response rate (ORR) was $28.0 \%$. In total, 58, 34, 19, and 18 patients had measurable hepatic tumors and lung, lymph node, and other intra-abdominal
\end{abstract}

Chih-Hung Hsu, MD, PhD or Ann-Lii Cheng, MD, PhD

Department of Oncology

National Taiwan University Hospita

7 Chung-Shan South Road, Taipei 10002 (Taiwan)

E-Mail chihhunghsu@ntu.edu.tw or alcheng@ ntu.edu.tw 


\section{Liver

metastases, and the corresponding organ-specific ORRs were 22.4, 41.2, 26.3, and 38.9\%, respectively. Of the 39 patients who had both hepatic and extrahepatic tumors, 12 had disease control in extrahepatic tumors while progressive disease (PD) in hepatic tumors, whereas only 4 exhibited disease control in hepatic tumors while PD in extrahepatic tumors $(p=0.046$, McNemar test). Of the 16 patients with only evaluable tumors in the liver and lungs at baseline, 8 had disease control in the lungs while PD in the liver, and none experienced disease control in the liver while PD in the lungs $(p=0.005)$. Conclusions: The hepatic tumors of HCC may be less responsive to $\mathrm{ICl}$ s than extrahepatic lesions. Lung metastases responded most favorably to ICls. The mechanisms underlying this differential response to ICls warrant further investigation.

(c) 2019 S. Karger AG, Basel

\section{Introduction}

Significant progress has been recently made in systemic therapy for advanced hepatocellular carcinoma (HCC), for which the only indicated therapy was sorafenib before 2017 [1, 2]. Several phase III trials have demonstrated the significant survival benefits of regorafenib, lenvatinib, and carbozantinib in patients with advanced HCC [3-5]. Moreover, immunotherapy targeting immune checkpoints, especially programmed cell death protein 1 (PD-1)/ programmed death-ligand 1 (PD-L1) and the cytotoxic T lymphocyte-associated antigen 4 (CTLA-4), has demonstrated significant clinical activity in advanced HCC [6-8].

In a recent phase I/II clinical trial (CheckMate 040) testing nivolumab, an anti-PD-1 monoclonal antibody ( $\mathrm{mAb}$ ), in patients with advanced HCC with or without prior sorafenib treatment, the response rate was approximately $20 \%$, and the overall survival (OS) among patients who had undergone prior sorafenib treatment was approximately 15 months [7]. Another anti-PD-1 mAb, pembrolizumab, exhibited a similar response rate $(17 \%)$ in patients with HCC who had previously been treated with sorafenib in a phase II study (KEYNOTE-224) [8]. In light of these promising results, the US Food and Drug Administration granted accelerated approval to nivolumab and pembrolizumab for treating HCC in patients who had undergone prior sorafenib treatment in 2017 and 2018, respectively. Many other immune checkpoint inhibitors (ICIs), as monotherapy or in combination with other agents, are under active investigation for treating HCC.

Mixed responses have been occasionally observed in patients with solid tumors who received chemotherapy or molecular targeted therapy [9-11]. These mixed responses may have resulted from heterogeneous clonalities of tumor cells $[12,13]$. The mechanism of action of ICIs is distinct from those of conventional chemotherapy and molecular targeted therapy. ICIs induce antitumor effects by reactivating exhausted $\mathrm{T}$ cells and thus rejuvenating antitumor immunity. Therefore, the differential tumor microenvironments of various organs may potentially influence the therapeutic effect of ICIs. Several studies have reported that patients with melanoma or renal cell carcinoma (RCC) experience mixed responses (i.e., metastatic tumors in one organ enlarge while those in another organ ameliorate or remain stable) to various types of immunotherapy [14-18]. However, such mixed tumor responses have not been reported in patients with HCC who received targeted therapy or ICIs. The objective of this study was to evaluate whether tumor response to ICIs in HCC varies among organs.

\section{Patients and Methods}

Study Population and Variables

We reviewed patients who were diagnosed with Barcelona Clinic Liver Cancer stage C HCC and received ICIs at 3 medical centers in Taiwan, including the National Taiwan University Hospital (NTUH) and Taipei 


\section{Liver Cancer}

Table 1. Baseline patient characteristics

\begin{tabular}{|c|c|}
\hline \multicolumn{2}{|l|}{ Liver Cancer 2019;8:480-490 } \\
\hline DOI: $10.1159 / 000501275$ & $\begin{array}{l}\text { (c) } 2019 \text { S. Karger AG, Basel } \\
\text { www.karger.com/lic }\end{array}$ \\
\hline
\end{tabular}

\begin{tabular}{lc}
\hline Total & $75(100.0)$ \\
Median age, years & $59.0(28.1-87.5)$ \\
Sex & $62(82.7)$ \\
$\quad$ Male & $13(17.3)$ \\
$\quad$ Female & \\
Child-Pugh class & $68(90.7)$ \\
A & $7(9.3)$ \\
B & $51(68.0)$ \\
HBsAg reactive & $11(14.7)$ \\
Anti-HCV reactive & \\
AFP, ng/mL & $41(54.7)$ \\
$\quad$ 400 & $34(45.3)$ \\
$\quad<400$ & \\
Prior systemic therapy & $53(70.7)$ \\
Sorafenib & $20(26.7)$ \\
None & \\
Disease sites ${ }^{1}$ & $58(77.3)$ \\
Liver & $42(56.0)$ \\
Lung & $20(26.7)$ \\
Lymph nodes & $19(25.3)$ \\
Intra-abdomen & $15(20.0)$ \\
Bone & \\
Immunotherapy & $56(74.7)$ \\
Anti-PD-1 mAb & $2(2.7)$ \\
Anti-PD-L1 mAb & $1(1.3)$ \\
Anti-CTLA-4 mAb & $16(21.3)$ \\
Anti-PD-1 plus anti-CTLA-4 mAb & \\
\hline
\end{tabular}

Data are presented as $n(\%)$ or median (range). HBsAg, hepatitis virus B surface antigen; anti-HCV, hepatitis C virus antibody; AFP, $\alpha$-fetoprotein; mAb, monoclonal antibody.

${ }^{1}$ In total, 58, 34, 19, and 18 patients had measurable hepatic tumors and lung, lymph node, and other intra-abdominal metastases at baseline.

Veterans General Hospital in Taipei and National Cheng Kung University Hospital in Tainan, between August 2015 and June 2017. Patients with advanced HCC who received either an anti-PD1/PD-L1 mAb, antiCLTA-4 $\mathrm{mAb}$, or a combination of these $2 \mathrm{mAbs}$ were identified and reviewed. Only patients who had measurable diseases and had subsequent image studies available for response evaluation were enrolled.

Clinical data were retrieved from the patients' medical records. The OS of the patients from the first ICI prescription date to either the date of death or the final follow-up date (December 31, 2017) was calculated. The data of patients who survived were censored. The time to treatment discontinuation (TTD) of the patients from the first ICI prescription date to the end date of the final treatment cycle was measured. This study was approved by the Research Ethics Committee of NTUH.

\section{Response Evaluation}

Before the ICI treatment was discontinued, the enrolled patients had undergone imaging studies for tumor response assessments every 6-12 weeks. Organ-specific response criteria, adapted from Response Evaluation Criteria in Solid Tumors (RECIST) 1.1 and immune-related RECIST (irRECIST), were established to evaluate the heterogeneous responses of different organ systems to immunotherapy [19, 20]. In brief, in each organ system, measurable lesions were defined as lesions with a longest diameter $\geq 1.0 \mathrm{~cm}$ and lymph nodes with short axis diameter $\geq 1.5 \mathrm{~cm}$. At baseline, a maximum of 5 lesions were identified as target lesions in each organ. Lesions of each organ were measured unidimensionally. The organ-specific complete responses (CR; complete disappearance or nodal short axis diameter $<1.0 \mathrm{~cm}$ on follow-up), partial responses (PR; $\geq 30 \%$ reduction), progressive diseases (PD; $\geq 20 \%$ increase), or stable diseases (SD; neither CR, PR, nor PD) 
Fig. 1. Kaplan-Meier curves of OS and TTD of patients with advanced HCC who received ICIs.

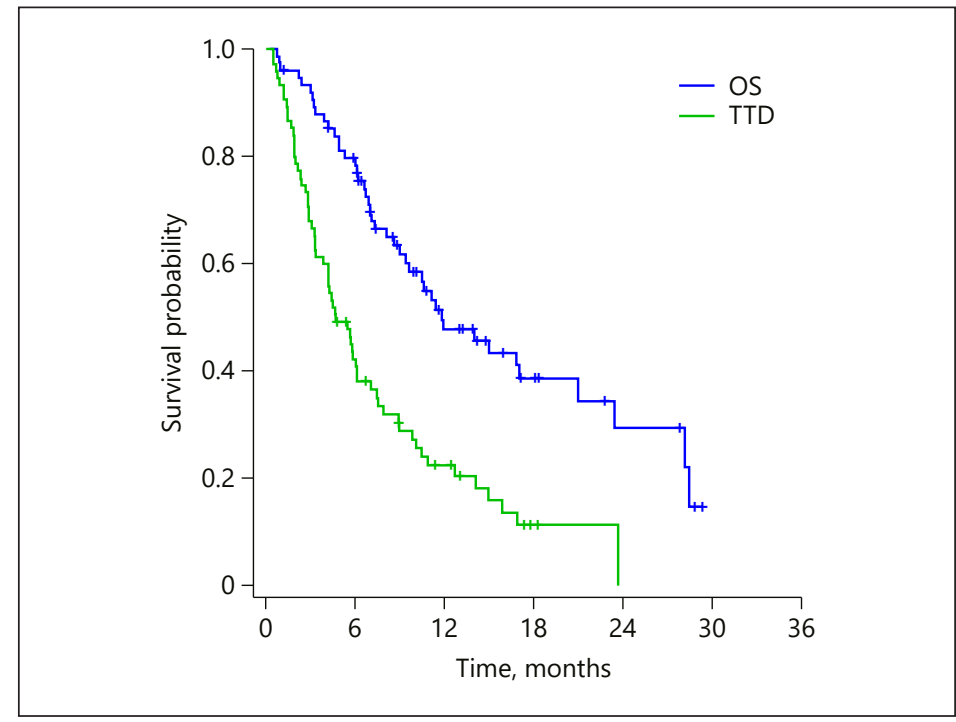

were determined for each organ system. New lesions did not always indicate PD. The dimensions of any new lesions were added to those of the original target lesions to determine the total tumor burden [20]. The best response of the lesions in each organ during the study period was recorded. Confirmation of CR, PR, or PD was not mandatory but was obtained if subsequent assessments were performed. An independent radiologist (I.-L.S.) who was blinded to the clinical data reviewed the pretreatment and posttreatment images and measured the best response of lesions in each organ system.

Statistical Analyses

Statistical analyses were performed using SAS statistical software (version 9.4, SAS Institute Inc., Cary, NC, USA). The OS and TTD of patients were estimated through the Kaplan-Meier method. The differential responses between different organ systems were compared using the McNemar test. The survival between patients with or without differential responses was compared by using the log-rank test. Two-sided $p$ values $<0.05$ were considered statistically significant.

\section{Results}

\section{Patient Characteristics}

We identified 95 patients with advanced HCC who received ICIs between August 2015 and June 2017. Among them, 16 patients who did not have subsequent imaging assessments to evaluate tumor responses and 4 patients who received ICIs plus other targeted therapy were excluded. The remaining 75 patients were included in this retrospective analysis. The baseline characteristics of the included patients are listed in Table 1. The median age was 59.0 years, and the majority were male $(82.7 \%)$ and had Child-Pugh class A liver reserve $(90.7 \%)$. Furthermore, $51(68.0 \%)$ and $11(14.7 \%)$ patients had chronic hepatitis B virus (HBV) or chronic hepatitis $\mathrm{C}$ virus infection, respectively. Overall, 20 patients $(26.7 \%)$ received ICIs as first-line treatment, and 53 patients $(70.7 \%)$ had received prior sorafenib treatment. Regarding ICI treatment, 56, 2, 1, and 16 patients received anti-PD-1 mAb, anti-PD-L1 mAb, anti-CTLA4 mAb, and anti-PD-1 mAb plus anti-CTLA4 mAb, respectively.

\section{Treatment Efficacy and Organ-Specific Responses}

The objective response rate (ORR) according to RECIST 1.1 was $28.0 \%$. The median OS was 11.8 months (range 0.7-29.3). The median TTD was 4.7 months (range 0.5-23.6; Fig. 1). 
Lu et al.: Organ-Specific Response to ICls in HCC

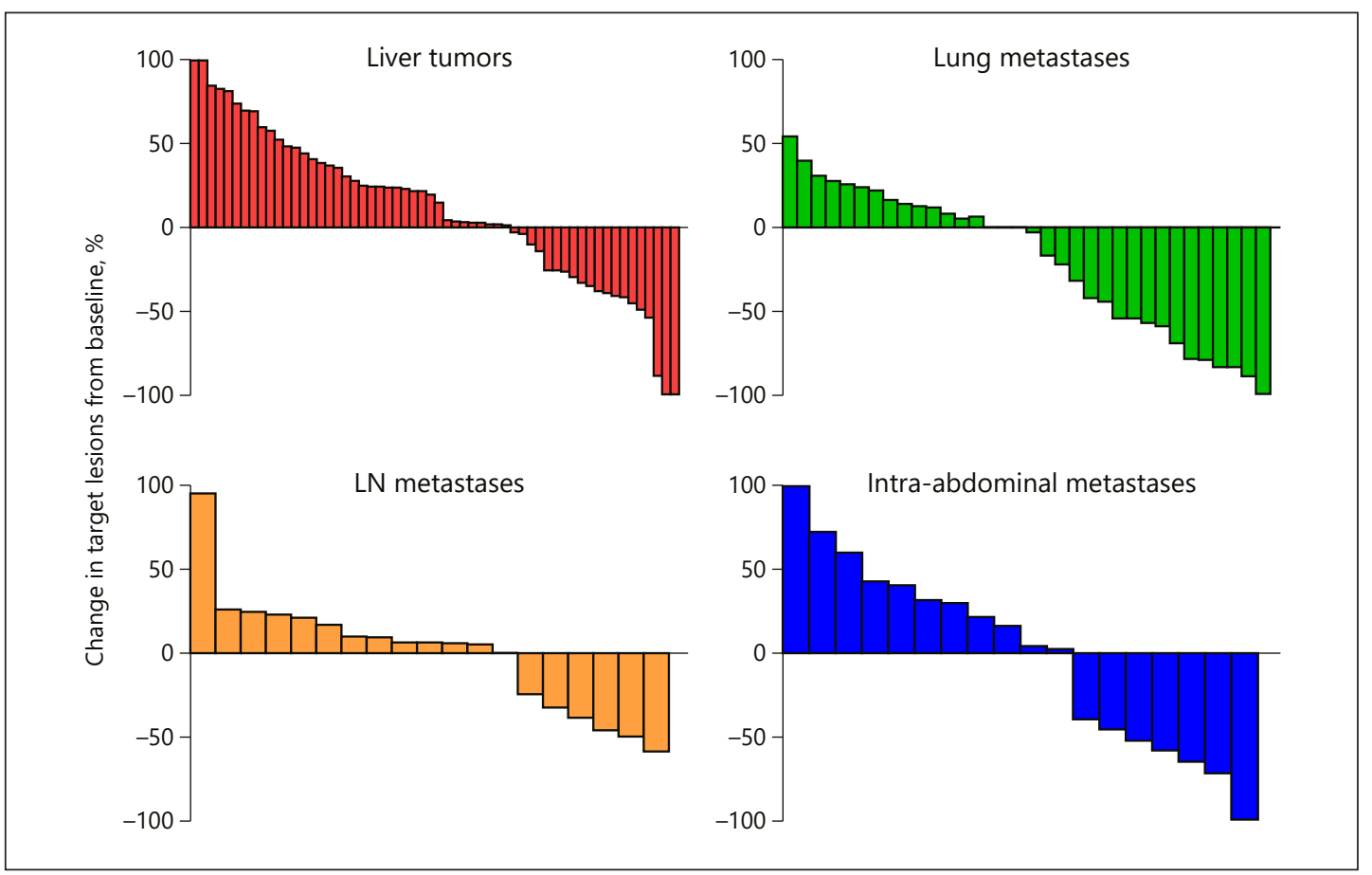

Fig. 2. Best percentage change over time (from baseline) in tumor burden in various organ systems.

Table 2. Median of mean tumor sizes and organ-specific responses to ICIs in HCC

\begin{tabular}{lcccc}
\hline & $\begin{array}{l}\text { Liver } \\
(n=58)\end{array}$ & $\begin{array}{l}\text { Lung } \\
(n=34)\end{array}$ & $\begin{array}{l}\text { Lymph node } \\
(n=19)\end{array}$ & $\begin{array}{l}\text { Intra-abdomen } \\
(n=18)\end{array}$ \\
\hline Median tumor size, cm (range) $^{1}$ & $2.4(1.0-15.9)$ & $1.6(1.0-8.6)$ & $2.1(1.5-8.8)$ & $2.6(1.0-7.7)$ \\
Objective response, \% & 22.4 & 41.2 & 26.3 & 38.9 \\
Complete response, $n(\%)^{\text {Partial response, } n(\%)}$ & $2(3.4)$ & $1(2.9)$ & 0 & $1(5.6)$ \\
Stable disease, $n(\%)$ & $11(19.0)$ & $13(38.2)$ & $5(26.3)$ & $6(33.3)$ \\
Progressive disease, $n(\%)$ & $16(26.7)$ & $13(38.2)$ & $9(47.4)$ & $3(16.7)$ \\
\hline
\end{tabular}

${ }^{1}$ In total, 150, 116, 37, and 40 hepatic tumors and lung, lymph node, and intra-abdominal metastases were measured, respectively.

As of the final follow-up date (December 31, 2017), 13 patients remained on ICI treatment, and 11 of these patients exhibited ongoing tumor responses.

Overall, 58, 34, 19, and 18 patients had measurable hepatic tumors and lung, lymph node, and other intra-abdominal metastases at baseline, respectively; these patients were subject to organ-specific response evaluation (Table 1). Regarding intra-abdominal metastases, 10, 5, 2, and 1 patients had peritoneal, adrenal, splenic, and subphrenic metastases, respectively. The median size of the tumors measured in hepatic tumors and lung, lymph node, and intra-abdominal metastases was 2.4, 1.6, 2.1, and $2.6 \mathrm{~cm}$, respectively. Organ-specific ORRs of hepatic tumors and lung, lymph node, and intra-abdominal metastases were $22.4,41.2,26.3$, and 38.9\%, respectively (Table 2). The median time to response of hepatic tumors and lung, lymph node, and intraabdominal metastases were 4.1, 4.1, 2.6, and 3.5 months, respectively. Figure 2 presents the best percentage change in tumor burden relative to that at baseline in different organ systems. 


\section{Liver

Lu et al.: Organ-Specific Response to ICls in HCC

The majority of patients (68\%) were HBV surface antigen reactive. For patients with HBV-related HCC $(n=49)$, the organ-specific ORRs of hepatic tumors, lung, lymph node, and intra-abdominal metastases were $23.7,38.5,23.1$, and $44.4 \%$, respectively, which were similar to those of the overall cohort of 75 patients. In terms of regimens of ICI, 16 patients received combination therapy with anti-PD-1 mAb plus anti-CTLA-4-mAb. After the exclusion of these 16 patients, the remaining 59 patients who received monotherapy had organ-specific ORRs of $14.6,40.0,18.8$, and $25.0 \%$ in liver, lung, lymph node, and intra-abdominal metastases, respectively. This pattern of response rates, with the lowest response rate in liver and highest in lung, was comparable to that of the overall cohort of 75 patients.

\section{Patients with Organ-Specific Differential Responses}

At baseline, 39 patients had both hepatic and extrahepatic tumors. Among them, 16 $(41.0 \%)$ exhibited differential responses to ICI treatment; 12 patients had disease control (CR, PR, or SD) in extrahepatic tumors while PD in hepatic tumors, whereas only 4 patients exhibited disease control in hepatic tumors and PD in extrahepatic tumors $(p=0.046$, McNemar test; online suppl. Table S1; see www.karger.com/doi/10.1159/000501275 for all online suppl. material). The baseline median hepatic tumor size was smaller in those 12 patients with PD in the liver $(2.0 \mathrm{~cm}$, range $1.0-15.0)$ than those 4 patients with PD in extrahepatic sites $(3.0 \mathrm{~cm}$, range 1.1-8.1). Among the 39 patients with both hepatic and extrahepatic tumors at baseline, the median OS in those with differential responses $(n=16)$ and those without $(n=23)$ was 7.3 versus 10.5 months, respectively ( $p=0.301$, log-rank test). Moreover, in 16 patients with only evaluable hepatic tumors and lung metastases at baseline, 8 had disease control in lung metastases while PD in hepatic tumors, whereas none experienced disease control in liver tumors and PD in lung metastases ( $p=0.005$; online suppl. Table S2). Figure 3 shows representative cases of such differential responses to ICI treatment.

\section{Discussion}

We retrospectively analyzed the data of 75 patients with advanced HCC who received ICIs and found that the tumor responses to ICIs varied significantly depending on the tumor site. Specifically, we found a favorable tumor response in lung metastatic lesions and a less favorable tumor response in hepatic tumors.

The heterogeneous tumor-immune microenvironments of various organs and the distinct gene expression profiles of various tumors of different patients may influence tumor growth as well as the responses to anticancer treatment $[21,22]$. Although a previous study exploring tumor-infiltrating immune cells, including CD8-positive T cells and DC-LAMP-positive dendritic cells, reported that the densities of these immune cells were similar in the primary tumors and pulmonary metastases of colorectal cancer and RCC [23], other studies have demonstrated different immune contextures, such as CD8-positive T cell density or PD-L1 expression level, in paired primary and metastatic RCC and lung cancer specimens [24, 25]. In our recent study, we found that PD-L1 expression in HCC tissues was not significantly higher in metastatic HCC tissues than in primary HCC tissues (online suppl. Fig. S1) [26]. Additional studies are required to comprehensively characterize the genomic, transcriptomic, and immunologic features of HCC tissues in different organs.

Mixed responses to immunotherapies other than ICIs have been observed in other solid tumors, mainly in melanoma [14-17]. In a subgroup of patients with metastatic melanoma treated with pembrolizumab in the KEYNOTE-001 phase I clinical trial, individual lesions were analyzed; lung lesions were found to have the highest rate of CR (42.3\%), followed by peritoneal (37.3\%) and liver (24.4\%) metastatic lesions [27]. In another study analyzing 


\section{Liver Cancer}

\begin{tabular}{l}
\hline Liver Cancer 2019;8:480-490 \\
\hline DOI: 10.1159/000501275
\end{tabular}

www.karger.com/lic

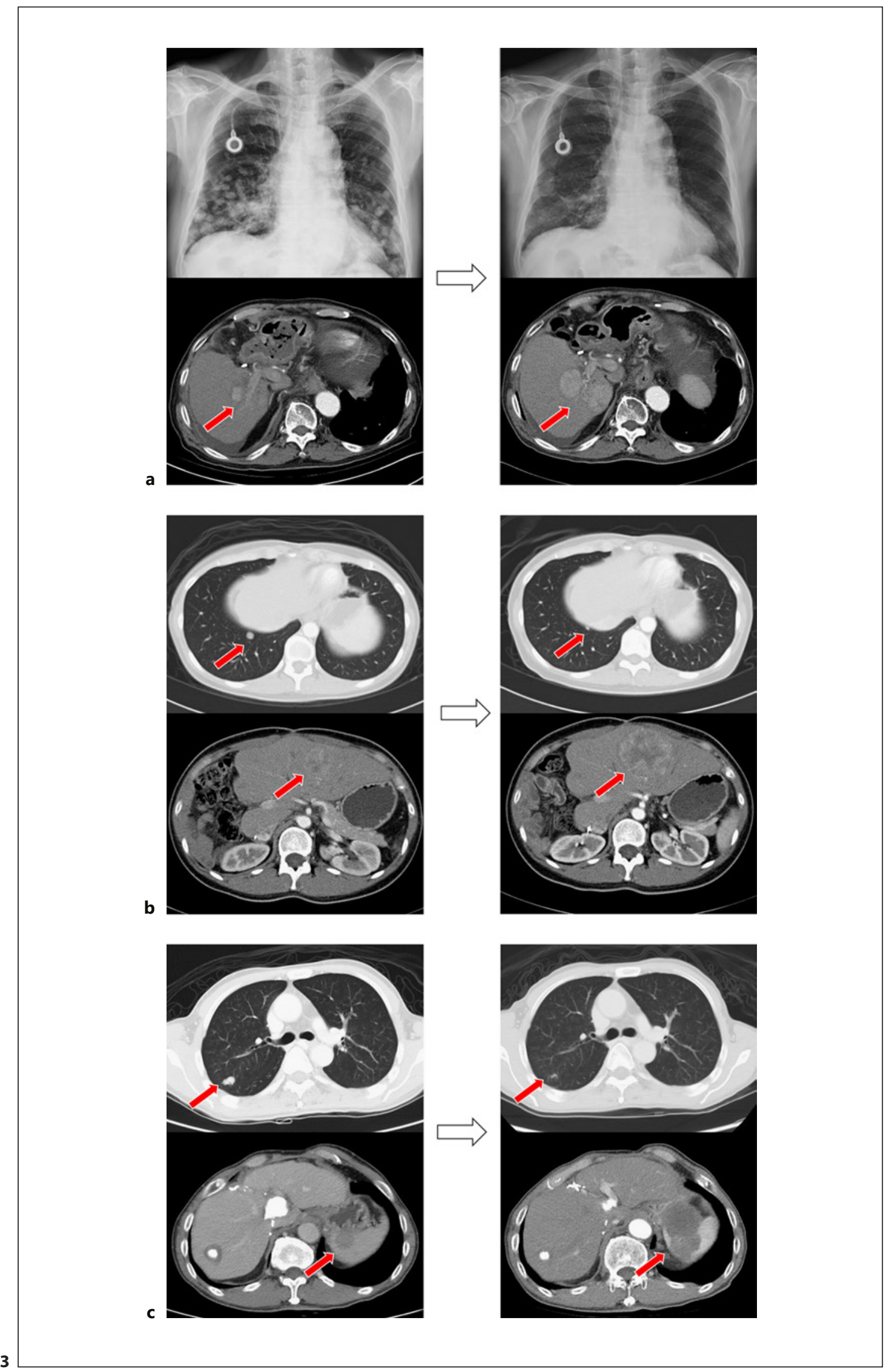




\section{Liver

patients with melanoma or non-small cell lung cancer (NSCLC) who received pembrolizumab in several clinical trials, decreased response rates and shortened progression-free survival were observed in patients with melanoma and NSCLC with liver metastases compared with those without liver metastases [28]. The response patterns reported in these studies are compatible with that in our study for HCC.

The liver is a unique organ that is constantly exposed to various antigens released from the gastrointestinal tract. Consequently, many cellular or molecular mechanisms are involved in maintaining the tolerogenic microenvironment in the liver $[29,30]$. For example, preclinical and clinical studies have demonstrated that the liver-resident macrophages, Kupffer cells, have been shown to induce T cell suppression [31,32]. Other immunosuppressive cells, such as regulatory $\mathrm{T}$ cells, tumor-associated macrophages, myeloid-derived suppressive cells (MDSCs), and hepatic stellate cells and hepatocytes, together render an immunosuppressive microenvironment in the liver [30]. A previous study showed that for HCC patients who received systemic chemotherapy, the ORRs of patients without intrahepatic disease tended to be higher than those of patients with active intrahepatic disease [33]. In our recent preclinical study, we found an increase in MDSCs and other immune suppressors in orthotopic liver tumors that progressed after sorafenib treatment, but not in subcutaneous tumors; in addition, targeting MDSCs with anti-Ly6G antibody enhanced T cell proliferation and the therapeutic effect of sorafenib in orthotopic liver tumors [34]. How to overcome the unfavorable immunosuppressive mechanisms in the microenvironment of the liver to improve the efficacy of immunotherapy warrants future investigation.

Imbalance between pretreatment tumor burden and $\mathrm{T}$ cell reinvigoration may lead to treatment failure of ICIs [35]. Based on the data of patients with melanoma who received pembrolizumab in the Keynote 001 phase I clinical trial, smaller tumors at baseline were associated with higher ORR and longer OS [36]. In another phase I study testing avelumab, an anti-PD-L1 mAb, in patients with metastatic urothelial carcinoma after failure of platinum chemotherapy, exploratory subgroup analyses also suggested that patients with smaller tumors at baseline had higher ORRs [37]. In our study, the median size of the measured hepatic tumors was numerically higher than that of the measured lung lesions ( $2.4 \mathrm{vs} .1 .6 \mathrm{~cm})$, and the organ-specific response to ICIs was lower for hepatic tumors than for lung lesions (22.4 vs. $41.2 \%$ ). However, intra-abdominal lesions, which had the largest median tumor size $(2.6 \mathrm{~cm})$ in our study, also exhibited a favorable organ-specific ORR (38.9\%). Additional studies are warranted to clarify whether tumor burden affects the efficacy of ICI treatment in HCC and other cancers.

Our study had limitations. First, our study was retrospective in nature and the sample size of those with both hepatic and extrahepatic tumors was small. Our findings need validation in prospective and large studies. Second, we did not employ modified RECIST (mRECIST) to evaluate hepatic tumor responses, thus ORR in liver tumors might be underestimated [38]. However, we consider mRECIST is more suitable for evaluating HCC patients who received antiangiogenic therapy, and most recent clinical trials testing ICIs in HCC use RECIST 1.1 or irRECIST irrespective of tumor location. Third, the patients in our study were heterogeneous in terms of regimens of ICIs received, dosing schedule, prior systemic therapy, and frequency of tumor assessment. Whether these factors influence the organ-specific differ-

Fig. 3. Representative images showing differential responses in different organ systems. a, b Two patients had regressed lung metastases but progressed hepatic tumors after treatment with ICIs. c Another patient had regressed lung metastases but progressed splenic metastatic tumor after treatment with ICIs. Left and right panels display image studies performed before and after ICI treatment, respectively. Arrows point to tumor sites with differential responses. 
Lu et al.: Organ-Specific Response to ICls in HCC

ential responses to ICIs is unclear and can only be addressed in a study with a more homogenous cohort. Finally, the mechanisms of organ-specific differential responses to ICIs were not explored in our study. It is inherently difficult to collect tumor samples from multiple organs of the same individual, especially simultaneously. Employing different preclinical models may help, at least in part, to determine the potential cellular and molecular mechanisms.

In conclusion, we found organ-specific differential responses to ICIs in patients with advanced HCC. HCC in the liver may be less responsive to ICIs than were extrahepatic metastases. Lung metastases exhibited the best response to ICIs. The underlying mechanism warrants further investigation.

\section{Acknowledgments}

This study was supported by grants from the Ministry of Science and Technology, Taiwan (MOST 106-2314-B-002-210, MOST 107-2314-B-002-188). The authors acknowledge the statistical assistance provided by the Department of Medical Research, NTUH. A substantial number of patients included in the current report were subjects enrolled in the CheckMate 040 study (NCT01658878). The authors are grateful to the study team of CheckMate 040 for supporting this analysis.

\section{Statement of Ethics}

This study was approved by the Research Ethics Committee of NTUH (201712219RINB). Written informed consent was obtained from the patients involved in the study.

\section{Disclosure Statement}

C.-H.H.: consulting or advisor role for Merck Serono, Novartis, Bristol-Myers Squibb, and Ono Pharmaceutical Co. Ltd, receiving honorarium from Bristol-Myers Squibb and Bayer. A.-L.C.: consultant of Novartis, Eisai, Merck Sharp \& Dohme (I.A.) Corp., Bayer, Bristol-Myers Squibb, and BeiGene, Ltd.

\section{References}

1 Llovet JM, Ricci S, Mazzaferro V, Hilgard P, Gane E, Blanc JF, et al.; SHARP Investigators Study Group. Sorafenib in advanced hepatocellular carcinoma. N Engl J Med. 2008 Jul;359(4):378-90.

2 Cheng AL, Kang YK, Chen Z, Tsao CJ, Qin S, Kim JS, et al. Efficacy and safety of sorafenib in patients in the AsiaPacific region with advanced hepatocellular carcinoma: a phase III randomised, double-blind, placebocontrolled trial. Lancet Oncol. 2009 Jan;10(1):25-34.

3 Bruix J, Qin S, Merle P, Granito A, Huang YH, Bodoky G, et al.; RESORCE Investigators. Regorafenib for patients with hepatocellular carcinoma who progressed on sorafenib treatment (RESORCE): a randomised, doubleblind, placebo-controlled, phase 3 trial. Lancet. 2017 Jan;389(10064):56-66.

4 Kudo M, Finn RS, Qin S, Han KH, Ikeda K, Piscaglia F, et al. Lenvatinib versus sorafenib in first-line treatment of patients with unresectable hepatocellular carcinoma: a randomised phase 3 non-inferiority trial. Lancet. 2018 Mar;391(10126):1163-73.

5 Abou-Alfa GK, Meyer T, Cheng AL, El-Khoueiry AB, Rimassa L, Ryoo BY, et al. Cabozantinib in patients with advanced and progressing hepatocellular carcinoma. N Engl J Med. 2018 Jul;379(1):54-63.

6 Sangro B, Gomez-Martin C, de la Mata M, Iñarrairaegui M, Garralda E, Barrera P, et al. A clinical trial of CTLA-4 blockade with tremelimumab in patients with hepatocellular carcinoma and chronic hepatitis C. J Hepatol. 2013 Jul;59(1):81-8.

7 El-Khoueiry AB, Sangro B, Yau T, Crocenzi TS, Kudo M, Hsu C, et al. Nivolumab in patients with advanced hepatocellular carcinoma (CheckMate 040): an open-label, non-comparative, phase 1/2 dose escalation and expansion trial. Lancet. 2017 Jun;389(10088):2492-502.

8 Zhu AX, Finn RS, Edeline J, Cattan S, Ogasawara S, Palmer D, et al.; KEYNOTE-224 investigators. Pembrolizumab in patients with advanced hepatocellular carcinoma previously treated with sorafenib (KEYNOTE224): a non-randomised, open-label phase 2 trial. Lancet Oncol. 2018 Jul;19(7):940-52. 
Lu et al.: Organ-Specific Response to ICls in HCC

9 van Kessel CS, Samim M, Koopman M, van den Bosch MA, Borel Rinkes IH, Punt CJ, et al. Radiological heterogeneity in response to chemotherapy is associated with poor survival in patients with colorectal liver metastases. Eur J Cancer. 2013 Jul;49(11):2486-93.

10 Chen ZY, Zhong WZ, Zhang XC, Su J, Yang XN, Chen ZH, et al. EGFR mutation heterogeneity and the mixed response to EGFR tyrosine kinase inhibitors of lung adenocarcinomas. Oncologist. 2012;17(7):978-85.

11 Mete UK, Singh DV, Kakkar N. Sustained systemic response paralleled with ovarian metastasis progression by sunitinib in metastatic renal cell carcinoma: is this an anti-angiogenic potentiation of cancer? Urol Ann. 2015 Jul-Sep;7(3):387-90.

12 Goasguen N, de Chaisemartin C, Brouquet A, Julié C, Prevost GP, Laurent-Puig P, et al. Evidence of heterogeneity within colorectal liver metastases for allelic losses, mRNA level expression and in vitro response to chemotherapeutic agents. Int J Cancer. 2010 Sep;127(5):1028-37.

13 Schmid K, Oehl N, Wrba F, Pirker R, Pirker C, Filipits M. EGFR/KRAS/BRAF mutations in primary lung adenocarcinomas and corresponding locoregional lymph node metastases. Clin Cancer Res. 2009 Jul;15(14):4554-60.

14 Abdi EA, Petrik P. An unusual case of renal cancer showing mixed tumor response to interferon. Am J Med. 1987 Dec;83(6):1147-50.

15 Lindsey KR, Gritz L, Sherry R, Abati A, Fetsch PA, Goldfeder LC, et al. Evaluation of prime/boost regimens using recombinant poxvirus/tyrosinase vaccines for the treatment of patients with metastatic melanoma. Clin Cancer Res. 2006 Apr;12(8):2526-37.

16 Carrasco J, Van Pel A, Neyns B, Lethé B, Brasseur F, Renkvist N, et al. Vaccination of a melanoma patient with mature dendritic cells pulsed with MAGE-3 peptides triggers the activity of nonvaccine anti-tumor cells. J Immunol. 2008 Mar;180(5):3585-93.

17 Bol KF, Figdor CG, Aarntzen EH, Welzen ME, van Rossum MM, Blokx WA, et al. Intranodal vaccination with mRNA-optimized dendritic cells in metastatic melanoma patients. Oncolmmunology.2015 Apr;4(8):e1019197.

18 Koelzer VH, Rothschild SI, Zihler D, Wicki A, Willi B, Willi N, et al. Systemic inflammation in a melanoma patient treated with immune checkpoint inhibitors-an autopsy study. J Immunother Cancer. 2016 Mar;4(1):13.

19 Eisenhauer EA, Therasse P, Bogaerts J, Schwartz LH, Sargent D, Ford R, et al. New response evaluation criteria in solid tumours: revised RECIST guideline (version 1.1). Eur J Cancer. 2009 Jan;45(2):228-47.

20 Nishino M, Giobbie-Hurder A, Gargano M, Suda M, Ramaiya NH, Hodi FS. Developing a common language for tumor response to immunotherapy: immune-related response criteria using unidimensional measurements. Clin Cancer Res. 2013 Jul;19(14):3936-43.

21 Jiménez-Sánchez A, Memon D, Pourpe S, Veeraraghavan H, Li Y, Vargas HA, Gill MB, et al. Heterogeneous tumor-immune microenvironments among differentially growing metastases in an ovarian cancer patient. Cell. 2017;170:927-938.e20.

22 Ascierto ML, Makohon-Moore A, Lipson EJ, Taube JM, McMiller TL, Berger AE, et al. Transcriptional Mechanisms of Resistance to Anti-PD-1 Therapy. Clin Cancer Res. 2017 Jun;23(12):3168-80.

23 Remark R, Alifano M, Cremer I, Lupo A, Dieu-Nosjean MC, Riquet M, et al. Characteristics and clinical impacts of the immune environments in colorectal and renal cell carcinoma lung metastases: influence of tumor origin. Clin Cancer Res. 2013 Aug;19(15):4079-91.

24 Baine MK, Turcu G, Zito CR, Adeniran AJ, Camp RL, Chen L, et al. Characterization of tumor infiltrating lymphocytes in paired primary and metastatic renal cell carcinoma specimens. Oncotarget. 2015 Sep;6(28):24990-5002.

25 Müller P, Rothschild SI, Arnold W, Hirschmann P, Horvath L, Bubendorf L, et al. Metastatic spread in patients with non-small cell lung cancer is associated with a reduced density of tumor-infiltrating $\mathrm{T}$ cells. Cancer Immunol Immunother. 2016 Jan;65(1):1-11.

26 Lu LC, Lee YH, Chang CJ, Shun CT, Fang CY, Shao YY, et al. Increased Expression of Programmed Death-Ligand 1 in Infiltrating Immune Cells in Hepatocellular Carcinoma Tissues after Sorafenib Treatment. Liver Cancer. 2019 Mar;8(2):110-20.

27 Khoja L, Kibiro M, Metser U, Gedye C, Hogg D, Butler MO, et al. Patterns of response to anti-PD-1 treatment: an exploratory comparison of four radiological response criteria and associations with overall survival in metastatic melanoma patients. Br J Cancer. 2016 Nov;115(10):1186-92.

28 Tumeh PC, Hellmann MD, Hamid O, Tsai KK, Loo KL, Gubens MA, et al. Liver Metastasis and Treatment Outcome with Anti-PD-1 Monoclonal Antibody in Patients with Melanoma and NSCLC. Cancer Immunol Res. 2017 May; 5(5):417-24.

29 Chan T, Wiltrout RH, Weiss JM. Immunotherapeutic modulation of the suppressive liver and tumor microenvironments. Int Immunopharmacol. 2011 Jul;11(7):879-89.

30 Tiegs G, Lohse AW. Immune tolerance: what is unique about the liver. J Autoimmun. 2010 Feb;34(1):1-6.

31 You Q, Cheng L, Kedl RM, Ju C. Mechanism of T cell tolerance induction by murine hepatic Kupffer cells. Hepatology. 2008 Sep;48(3):978-90.

32 Wu K, Kryczek I, Chen L, Zou W, Welling TH. Kupffer cell suppression of CD8+ T cells in human hepatocellular carcinoma is mediated by B7-H1/programmed death-1 interactions. Cancer Res. 2009 0ct;69(20):8067-75.

33 Ikeda M, Okusaka T, Ueno H, Morizane C, Kojima Y, Iwasa S, et al. Predictive factors of outcome and tumor response to systemic chemotherapy in patients with metastatic hepatocellular carcinoma. Jpn J Clin Oncol. 2008 Oct;38(10):675-82.

34 Chang CJ, Yang YH, Chiu CJ, Lu LC, Liao CC, Liang CW, et al. Targeting tumor-infiltrating Ly6G+ myeloid cells improves sorafenib efficacy in mouse orthotopic hepatocellular carcinoma. Int J Cancer. 2018 May;142(9): 1878-89. 
35 Huang AC, Postow MA, Orlowski RJ, Mick R, Bengsch B, Manne S, et al. T-cell invigoration to tumour burden ratio associated with anti-PD-1 response. Nature. 2017 May;545(7652):60-5.

36 Joseph RW, Elassaiss-Schaap J, Kefford R, Hwu WJ, Wolchok JD, Joshua AM, et al. Baseline Tumor Size Is an Independent Prognostic Factor for Overall Survival in Patients with Melanoma Treated with Pembrolizumab. Clin Cancer Res. 2018 Oct;24(20):4960-7.

37 Patel MR, Ellerton J, Infante JR, Agrawal M, Gordon M, Aljumaily R, et al. Avelumab in metastatic urothelial carcinoma after platinum failure (JAVELIN Solid Tumor): pooled results from two expansion cohorts of an open-label, phase 1 trial. Lancet Oncol. 2018 Jan;19(1):51-64.

38 Lencioni R, Llovet JM. Modified RECIST (mRECIST) assessment for hepatocellular carcinoma. Semin Liver Dis. 2010 Feb;30(1):52-60. 